\title{
Preferential CTL targeting of Gag is associated with relative viral control in long-term surviving HIV-1 infected former plasma donors from China
}

\author{
Mingming Jia ${ }^{1, *}$, Kunxue Hong ${ }^{1, *}$, Jianping Chen $^{1}$, Yuhua Ruan ${ }^{1}$, Zhe Wang $^{2}$, Bing $\mathrm{Su}^{3}$, Guoliang Ren ${ }^{1}$, \\ Xiaoqing Zhang ${ }^{1}$, Zhen Liu ${ }^{1}$, Quanbi Zhao ${ }^{1}$, Dan $\mathrm{Li}^{1}$, Hong Peng ${ }^{1}$, Marcus Altfeld ${ }^{4}$, Bruce D Walker ${ }^{4}, \mathrm{Xu} \mathrm{G} \mathrm{Yu}^{4}$, \\ Yiming Shao ${ }^{1}$ \\ ${ }^{1}$ State Key Laboratory for Infectious Disease Prevention and Control, National Center for AIDS/STD Control and Prevention, \\ Chinese Center for Disease Control and Prevention, Beijing 102206, China; ${ }^{2}$ Henan Center for Disease Control and Prevention, \\ Zhengzhou, Henan 450016, China; ${ }^{3}$ Anhui Center for Disease Control and Prevention, Hefei, Anhui 230061, China; ${ }^{4}$ Ragon Insti- \\ tute of MGH, MIT, and Harvard, Charlestown, MA 02129, USA
}

It is generally believed that $\mathrm{CD8}^{+}$cytotoxic $\mathrm{T}$ lymphocytes (CTLs) play a critical role in limiting the replication of human immunodeficiency virus type 1 (HIV-1) and in determining the outcome of the infection, and this effect may partly depend on which HIV product is preferentially targeted. To address the correlation between HIV-1-specific CTL responses and virus replication in a cohort of former plasma donors (FPDs), 143 antiretroviral therapy naive FPDs infected with HIV-1 clade B' strains were assessed for HIV-1-specific CTL responses with an IFN- $\gamma$ Elispot assay at single peptide level by using overlapping peptides (OLPs) covering the whole consensus clade B proteome. By using a Spearman's rank correlation analysis, we found that the proportion of Gag-specific CTL responses among the total virus-specific CTL activity was inversely correlated with viral loads while being positively correlated to CD4 counts, as opposed to Pol- and Env-specific responses that were associated with increased viral loads and decreased CD4 counts. In addition, Vpr-specifc CTL responses showed a similar protective effect with Gag responses, but with a much lower frequency of recognition. Significantly, we also observed an association between HLA-A*30/B*13/ $\mathrm{Cw}^{* 06}$ haplotype and lower viral loads that was probably due to restricted Gag-specific CTL responses. Thus, our data demonstrate the prominent role of Gag-specific CTL responses in disease control. The advantage of HLA-A*30/ $B * 13 / C^{*} * 6$ haplotype in viral control may be associated with the contribution of Gag-specific CTL responses in the studied individuals.

Keywords: human immunodeficiency virus type 1; cytotoxic T lymphocytes; human leukocyte antigen class I; Gag Cell Research (2012) 22:903-914. doi:10.1038/cr.2012.19; published online 31 January 2012

\section{Introduction}

A variety of studies have demonstrated the role of human leukocyte antigen class I (HLA-I)-restricted cytotoxic T lymphocyte (CTL) responses in driving and shaping human immunodeficiency virus type 1 (HIV-1)

\footnotetext{
*These two authors contributed equally to this work. Correspondence: Yiming Shao

Tel: +86-10-58900981; Fax: +86-10-58900980

E-mail: yshao08@gmail.com

Received 22 May 2011; revised 25 August 2011; accepted 31 October 2011; published online 31 January 2012
}

evolution in vivo as well as the associations of particular HLA genotypes with HIV transmission and disease progression to AIDS. This gives rise to the hypothesis that CTLs against particular epitopes restricted by different HLA molecules may differ in their ability to control HIV replication [1-4], with a superior role of Gag-specific responses [5-8]. Two main explanations for Gag's beneficial role in HIV-1 viral control include its early efficient expression, processing and presentation in the cell $[9,10]$ and functional constraints leading to a high fitness cost when escape mutations occur at certain regions $[11,12]$. However, there are also a number of studies questioning the role of Gag-specific CTL responses, but in most of 
these studies, association between viral control and CTLs was analyzed with total virus- or Gag-specific responses [13-15]. Recent studies associated the proportion of Gagspecific CTL responses among the total virus-specific CTL activity with viral control, while the breadth and magnitude of the total virus-specific CTL responses did not show this significant association $[6,8]$. Hence, characterizing virus-specific CTL responses at single peptide levels and addressing the correlation between CTL responses and virus control in a population that is genetically different from previously-studied Caucasian and African cohorts will extend efforts to identify the correlates of immune protection against HIV-1. Here we investigated the HIV-1-specific CTL responses against overlapping peptides (OLPs) covering the entire consensus HIV-1 clade B proteome using the interferon (IFN)- $\gamma$ Elispot assay in a former plasma donor (FPD) cohort of 143 individuals with chronic, untreated HIV-1 clade B' infection in China. Correlations between HIV-1 proteinspecific CTL responses and plasma viral loads or CD4 counts were calculated for each of the HIV-1 proteins.

\section{Results}

Frequent recognition of Gag in HIV-1 clade B' infected Chinese HIV-1 patients

CTL responses against the HIV-1 proteome were evaluated by using IFN- $\gamma$ Elispot assays. HIV-1 proteins were recognized with different frequencies by the study population (Figure 1). The structural HIV-1 proteins Gag, Pol, Env and the accessory protein Nef were targeted by $93.01 \%, 86.71 \%, 72.73 \%$ and $78.32 \%$ of studied individuals, respectively. The accessory proteins Vpr, Vpu, Vif and the regulatory proteins Tat and Rev represented minor targets for CTL and were recognized by $16.78 \%$, $11.19 \%, 29.37 \%, 33.57 \%$ and $32.87 \%$ of studied individuals, respectively. When analyzing CTL responses on a single peptide-specific level, we found that $92.54 \%$ of Gag OLPs were targeted at least once by HIV-1 specific CTLs; most of the other proteins were less intensely targeted (Table 1). To avoid the effect of the size of different proteins, the average recognition frequency of OLPs was calculated for each HIV-1 protein. The average recognition frequency of OLPs of HIV-1 proteins was in the order of Gag $>$ Nef $>$ Tat $>$ Rev $>$ Pol $>$ Vpr $>$ Vif $>$ Env $>$ Vpu (Table 1). Taken together, these data reflect the dominance of Gag-specific T-cell responses within this cohort.

Relative dominance of Gag-specific CTL responses is associated with immune control

To explore the correlation between CTL responses and immune control of HIV-1 infection, many studies directly compared viral loads (or CD4 counts) to the

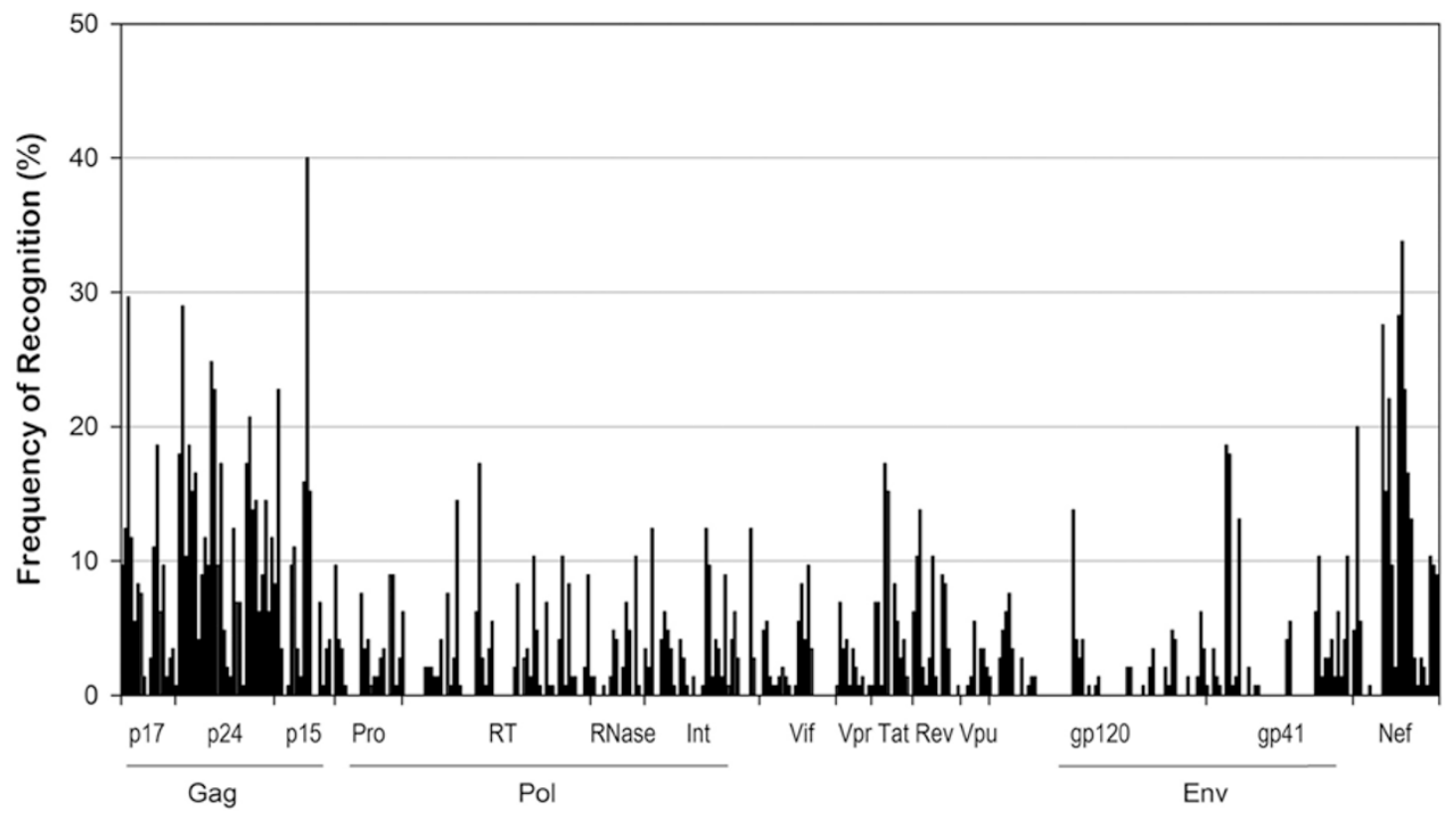

Figure 1 The overall CTL responses are distributed over the HIV proteome and cluster in immunodominant regions. A total of 143 FPDs infected with HIV-1 B' were tested by using an OLP set of 413 peptides. The figure shows the frequency of recognition for each single peptide among the 143 subjects tested ( $y$ axis). The words under $x$ axis indicate the HIV proteins spanned by the OLPs. 
Table 1 Distribution of CTL responses between HIV proteins

\begin{tabular}{|c|c|c|c|c|c|}
\hline HIV Protein & $\%$ of OLPs targeted & $\begin{array}{c}\% \text { of subjects with } \\
\text { positive response } \\
(n=143)\end{array}$ & $\begin{array}{l}\text { Average recognition } \\
\text { frequency of OLPs } \\
(\%)\end{array}$ & $\begin{array}{c}\text { Median of } \\
\text { positive response } \\
\left(\mathrm{SFC} / 10^{6} \mathrm{PBMC}\right)\end{array}$ & $\begin{array}{c}25 \%-75 \% \text { of } \\
\text { positive response } \\
\left(\mathrm{SFC} / 10^{6} \mathrm{PBMC}\right)\end{array}$ \\
\hline Pol & $67.67(90 / 133)$ & 86.71 & 2.90 & 672.5 & $292.5-1255$ \\
\hline Env & $48.25(55 / 114)$ & 72.73 & 1.93 & 461.3 & $207.1-970.4$ \\
\hline Nef & $81.48(22 / 27)$ & 78.32 & 9.76 & 515 & $270-1445$ \\
\hline $\mathrm{Vpu}$ & $66.67(6 / 9)$ & 11.19 & 1.86 & 193.8 & $141.3-596.3$ \\
\hline $\mathrm{Vpr}$ & $90.91(10 / 11)$ & 16.78 & 2.23 & 180 & $113.8-357.5$ \\
\hline Vif & $62.50(15 / 24)$ & 29.37 & 2.13 & 205 & $97.5-391.9$ \\
\hline
\end{tabular}

total breadth and magnitude of IFN- $\gamma$ responses against HIV-1 proteins, and controversial results were obtained $[13,14,16]$. More recently, several studies focused on the relative dominance of CTL targeting, and suggested that the preferential targeting of Gag but not the absolute value of Gag-specific CTL responses was associated with viral control $[6,17]$. In the present study, both the total breadth and magnitude of immune responses directed against individual OLPs spanning all HIV products and their relative contribution to the total virus-specific CTL activity were assessed and compared to viral loads as well as CD4 counts to get comparable data with other studies based on diverse cohorts, which maintain viral and host genetic heterogeneity.

First, we examined the correlation of CTL responses with viral loads using Spearman's rank order correlation analysis and found that there was a positive correlation between the cumulative magnitude of total HIV-specific CTL responses and viral loads $\left(r_{\mathrm{s}}=0.1926, P=0.0212\right)$. When the breadth of total responses was compared to viral loads, no significant correlation was found. The magnitude and breadth of Pol- and Env-specific CTL responses were found to be positively correlated with viral loads (Pol-magnitude: $r_{\mathrm{s}}=0.3255, P<0.0001$; Polbreadth: $r_{\mathrm{s}}=0.2618, P=0.0016$; Env-magnitude: $r_{\mathrm{s}}=$ $0.3785, P<0.0001$; Env-breadth: $r_{\mathrm{s}}=0.337, P<0.0001$ ) (Figures 2A and 3A, Supplementary information, Table $\mathrm{S} 1$ ), as well as the magnitude but not breadth of Nefspecific CTL responses (Nef-magnitude: $r_{\mathrm{s}}=0.1979, P=$ 0.0178). In addition, an inverse correlation between Vprspecific responses and viral loads was observed for both magnitude and breadth (Vpr-magnitude: $r_{\mathrm{s}}=-0.2001, P$ $=0.0165$; Vpr-breadth: $\left.r_{\mathrm{s}}=-0.2034, P=0.0148\right)$.

When correlation between IFN- $\gamma$ responses and CD4 counts was analyzed, a weak negative association between the Vpu-specific responses and CD4 counts was observed (Vpu-magnitude: $r_{\mathrm{s}}=-0.1694, P=0.0431$; Vpu-breadth: $\left.r_{\mathrm{s}}=-0.1691, P=0.0435\right)$. Furthermore, we found that an increased breadth of CTL responses targeting Vpr was associated with elevated CD4 counts (Vpr-breadth: $r_{\mathrm{s}}=0.1655, P=0.0483$ ). No other total or protein-specific responses were associated with CD4 counts (Supplementary information, Figures S1A, S2A and Table S1).

Similar to previous studies $[6,17]$, we next calculated the relative magnitude and relative breadth of proteinspecific responses by comparing the magnitude of protein-specific responses to the magnitude of total HIV1 -specific responses and by comparing the number of targeted OLPs in a protein to the total number of recognized OLPs, and then examined the association between viral loads as well as CD4 counts and the contribution of HIV-1 protein-specific responses to total responses. Interestingly, while overall Gag-specific responses were not associated with viral loads and CD4 counts, we observed a statistically significant inverse correlation between viral loads and the contribution of Gag-specific responses to the total magnitude of CTL responses (relative Gag-magnitude: $r_{\mathrm{s}}=-0.2874, P=0.0005$; relative Gag-breadth: $r_{\mathrm{s}}=-0.3139, P=0.0001$ ) (Figures $2 \mathrm{~B}$ and 3B, Supplementary information, Table S1). Moreover, we also found a statistically significant positive correlation between CD4 counts and the relative proportion of Gag-specific CTL responses (relative Gag-magnitude: $r_{\mathrm{s}}$ $=0.2442, P=0.0033$; relative Gag-breadth: $r_{\mathrm{s}}=0.1682$, $P=0.0447$ ) (Supplementary information, Figures S1B, S2B and Table S1). In contrast, comparison between the relative magnitude and breadth of Nef-specific responses and viral loads or CD4 counts did not yield significant associations though there was a direct correlation between viral loads and the magnitude of Nef-specific responses. The contribution of Pol- and Env-specific 


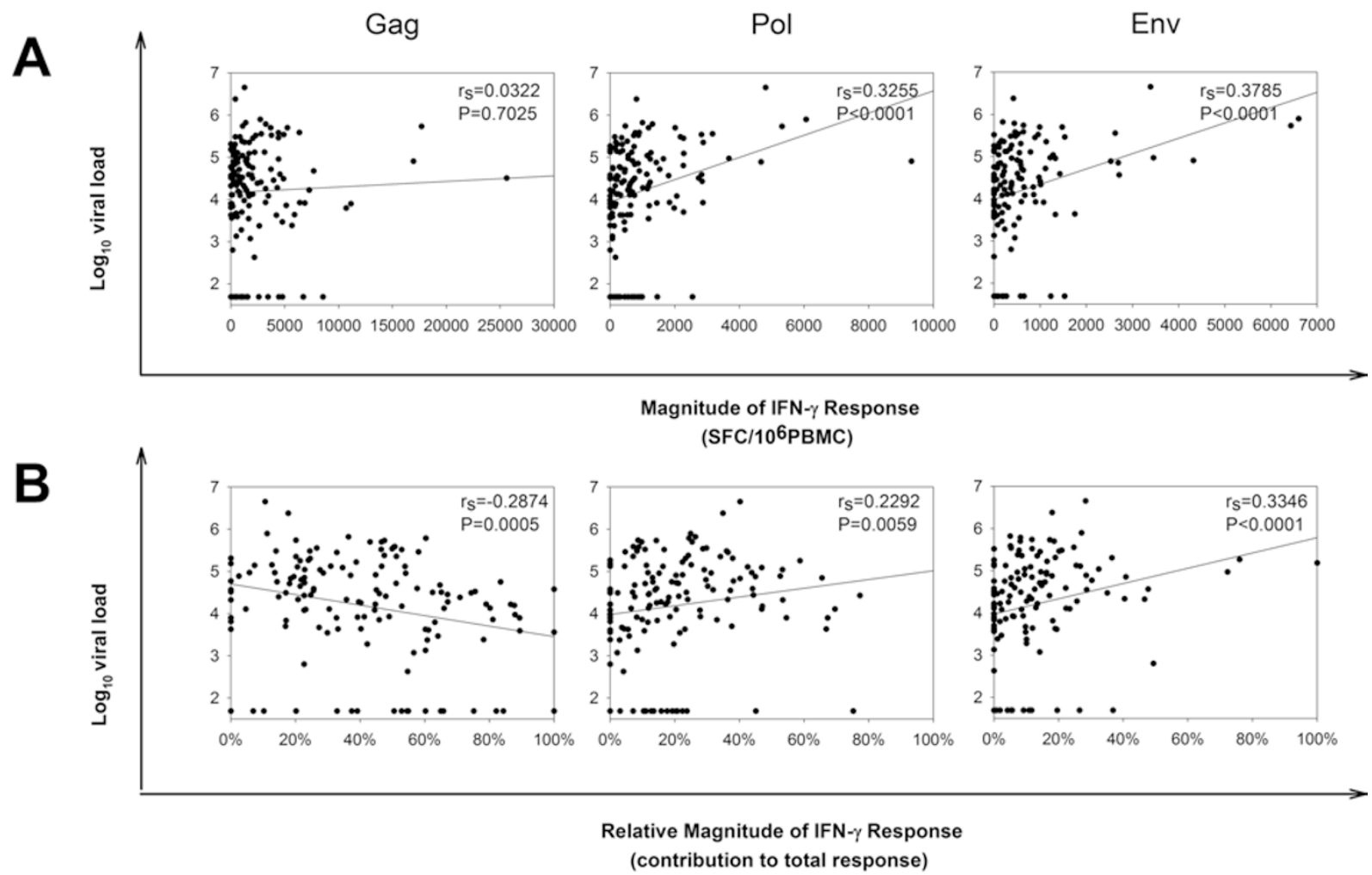

Figure 2 Correlation of the magnitude and contribution of HIV-1-specific CTL responses with plasma viral loads. (A) For the 143 untreated and chronically infected subjects, the magnitude of responses was compared to individuals' viral loads. (B) Relative magnitude was calculated for the proportion of the HIV-1 Gag-, Pol- and Env-specific response among the total response, and compared to individuals' viral loads. The numbers on the $y$ axis represent the $\log _{10}$ viral load. $P$-values (two-sided) and $r_{\mathrm{s}}$ values are based on Spearman's rank test. The solid lines represent the linear trend.

responses to total response was found to be positively correlated with viral loads (relative Pol-magnitude: $r_{\mathrm{s}}$ $=0.2292, P=0.0059$; relative Pol-breadth: $r_{\mathrm{s}}=0.2088$, $P=0.0123$; relative Env-magnitude: $r_{\mathrm{s}}=0.3346, P<$ 0.0001; relative Env-breadth: $r_{\mathrm{s}}=0.3311, P<0.0001$ ) (Figures $2 \mathrm{~B}$ and $3 \mathrm{~B}$, Supplementary information, Table S1), while for the contribution of Vpr-specific response to total response, an inverse correlation with viral loads (relative Vpr-magnitude: $r_{\mathrm{s}}=-0.2371, P=0.0043$; relative Vpr-breadth: $r_{\mathrm{s}}=-0.209, P=0.0123$ ) and a positive correlation with CD4 counts were found (relative Vprmagnitude: $r_{\mathrm{s}}=0.178, P=0.0334$; relative Vpr-breadth: $r_{\mathrm{s}}=0.1671, P=0.0461$ ) (Supplementary information, Table S1).

Since multiple Spearman's rank correlation analyses were performed in this study, we performed Benjamini and Hochberg False Discovery Rate procedure to reduce the overall false positive rate. Some of the comparisons (e.g., the correlations between Vpu-specific response and CD4 counts) lost the significance after correction of multiple testing (Supplementary information, Table S1).
However, the main findings such as the negative correlation with viral load of the relative dominance of Gagspecific CTLs as well as the positive correlations with viral load of CTLs against Pol and Env remain statistically significant under more stringent tests (Supplementary information, Table S1).

\section{HIV-1-specific CTL responses among elite controllers and AIDS patients}

Two extreme disease progression groups - HIV-1 elite controllers (viral load $<$ lower detection limit (LDL), CD4 count $\left.>500 / \mathrm{mm}^{3}, n=14\right)$ and AIDS patients (CD4 count $<200 / \mathrm{mm}^{3}, n=17$ ) were selected for detailed analysis of the relationship between HIV-induced CTL responses and disease progression (Figure 4A and Supplementary information, Table S2). When examining single OLP-specific CTL responses, we found that nine OLPs were targeted with significantly different frequencies between the two groups based on a Fisher's exact test. Among the nine OLPs, four Gag-OLPs, two VprOLPs and one Pol-OLP were significantly overrepresent- 

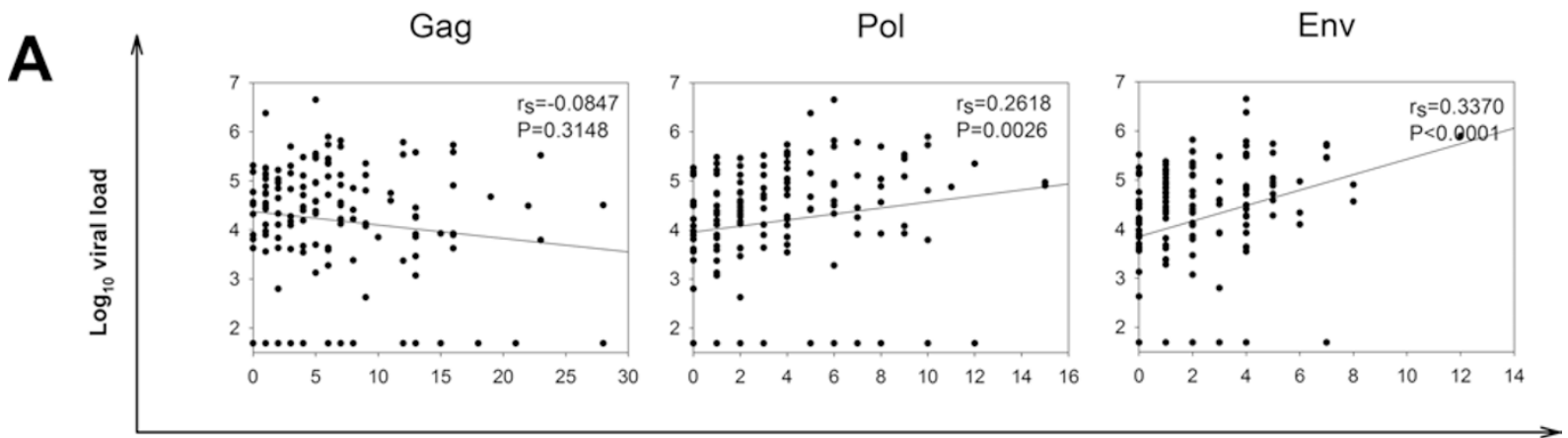

Breadth of IFN- $-\gamma$ Response
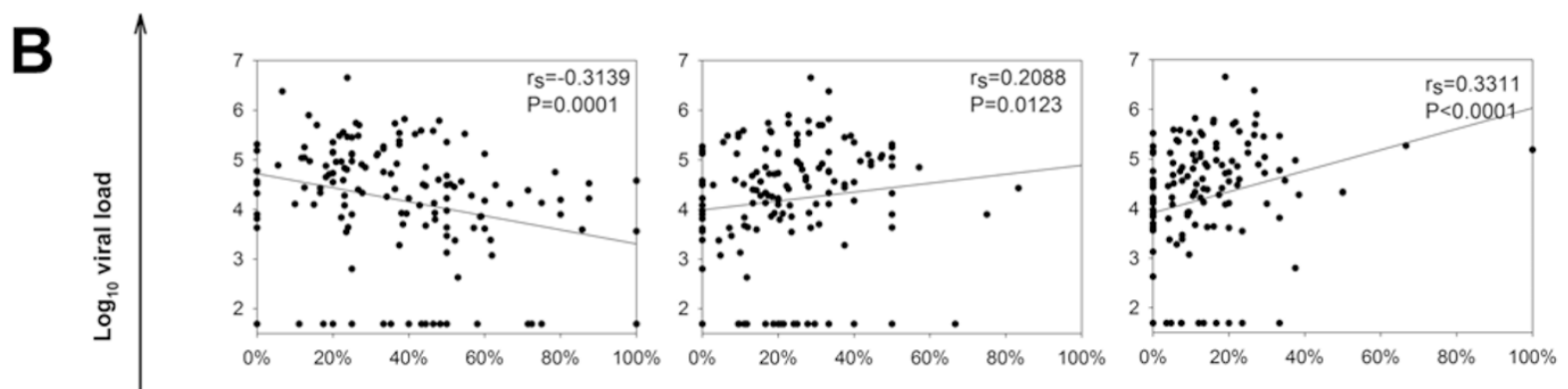

Relative Breadth of IFN- $\gamma$ Response

(contribution to total response)

Figure 3 Correlation of the breadth and contribution of HIV-1-specific CTL responses with plasma viral loads. (A) For the 143 untreated and chronically infected subjects, the breadth of responses was compared to individuals' viral loads. (B) Relative breadth was calculated for the proportion of the HIV-1 Gag-, Pol- and Env-specific response among the total response, and compared to individuals' viral loads. The numbers on the $y$ axis represent the $\log _{10}$ viral load. $P$-values (two-sided) and $r_{\mathrm{s}}$ values are based on Spearman's rank test. The solid lines represent the linear trend.

ed among the elite controllers (Table 2 and Figure 4B). With correction of multiple testing, all the significant differences of the nine OLPs disappeared $(P>0.05)$. However, considering that up to 413 peptides are used in the present study, multiple testing corrections might be too stringent. Of note, two of the four Gag-OLPs contained previously defined CTL epitopes, KRWIILGLNK (Gag residues 263-272) and ISPRTLNAW (Gag residues 147$155)$, the recognition of which has been repeatedly associated with slow HIV/AIDS progression. The recognition of these "good" OLPs may have an implication in the control of HIV/AIDS progression. It is worthy to check whether the other five OLPs also contain such "good" epitopes. Next, the level of HIV-1-specific CTL responses (evaluated by magnitude and breadth) and the contribution of each protein-specific CTL response among total responses (evaluated by relative magnitude and relative breadth) were compared between the two groups by using Mann-Whitney tests. There was no statistically significant difference between cumulative magnitude and breadth of the total HIV-specific CTLs between the two groups $(P>0.05)$. The Vpr-induced CTL response of elite controllers was significantly stronger compared to AIDS patients (Vpr-magnitude: $P=0.0049$, Vpr-breadth: $P=0.0048$ ) (Supplementary information, Table S2). Additionally, there was a significantly higher relative magnitude of Gag-specific responses in elite controllers compared to AIDS patients, while differences between the relative breadth of responses approached statistical significance (relative Gag-magnitude: $P=0.0033$, relative Gag-breadth: $P=0.0707$ ). We also found that the relative magnitude and relative breadth of Vpr-specific CTL responses induced in elite controllers were significantly higher than that of AIDS patients (relative Vprmagnitude: $P=0.0045$, relative Vpr-breadth: $P=0.0049$ ).

To address the relationship between the host HLA-I alleles and disease control, the HLA allele distribution among the two different disease progression groups was compared using a Fisher's exact test, and no statistically significant difference was found. Of note, the number of subjects enrolled in this analysis is relatively small, which may have significantly influenced the statistical 
A

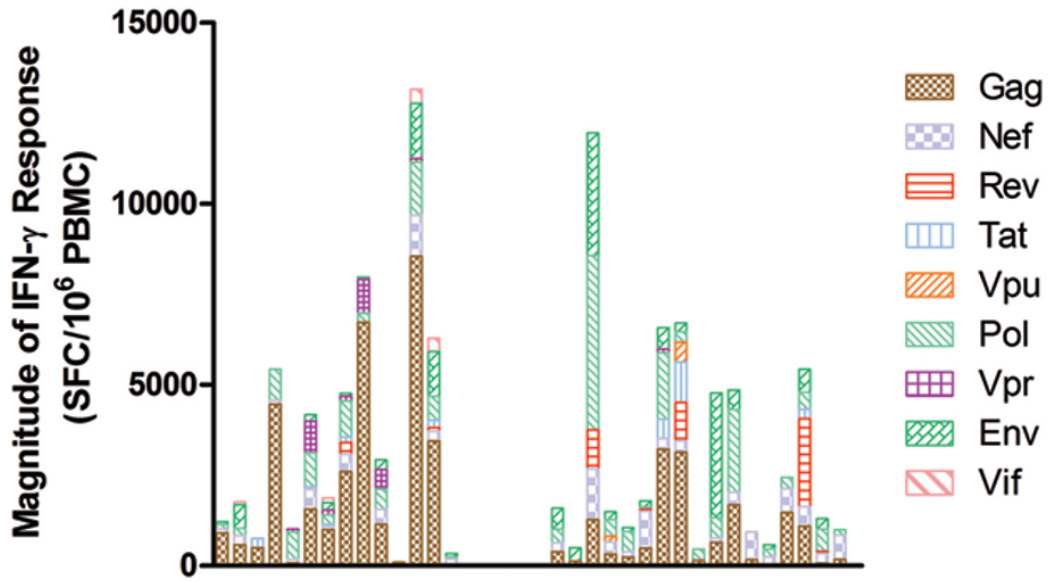

\section{Elite Controllers AIDS Patients}

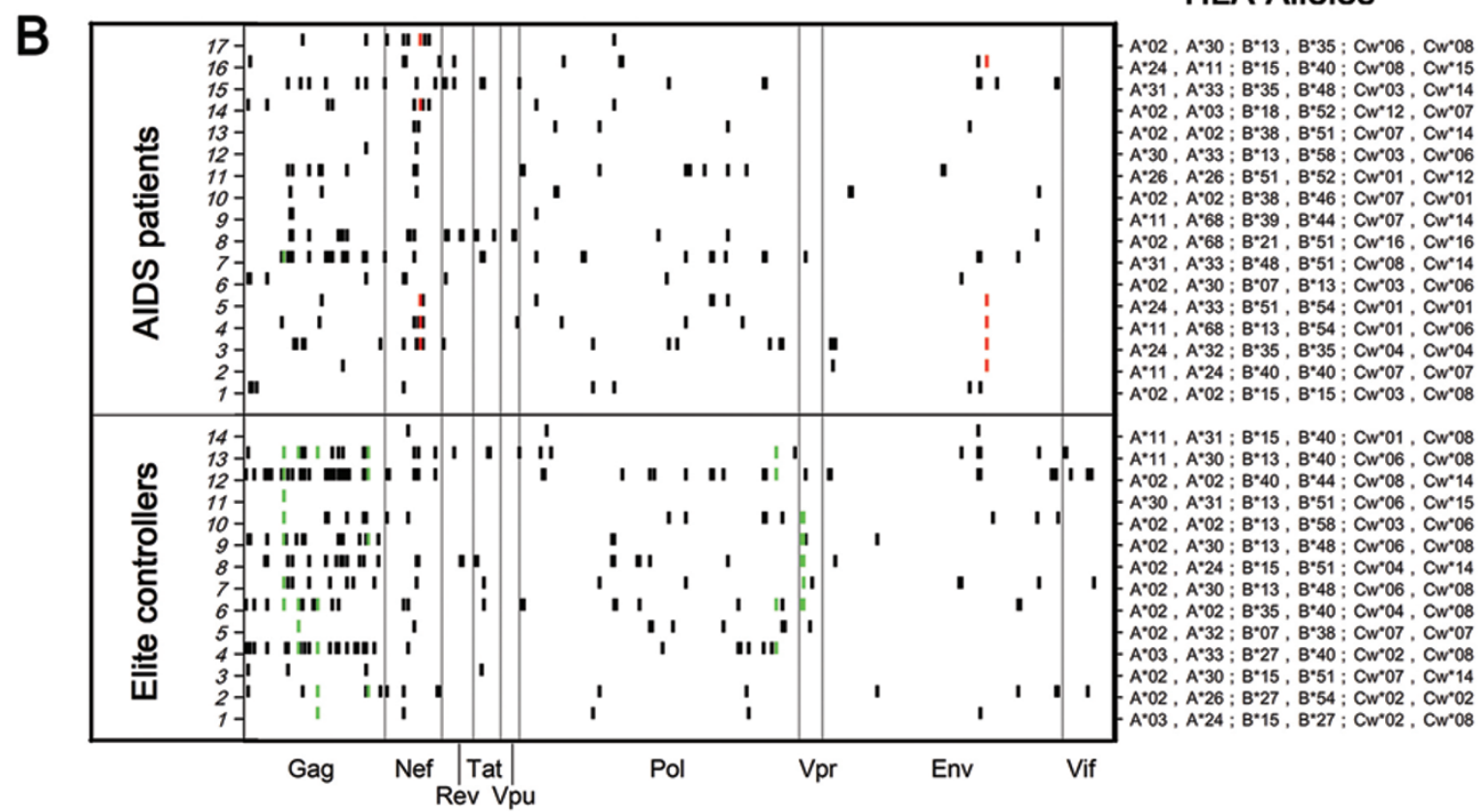

Figure 4 Distribution of HIV-1-specific CTL responses among different disease progression groups. (A) Distribution of HIV-1specific CTL responses within 14 elite controllers and 17 AIDS patients to nine HIV-1 proteins. CTL responses to each HIV1 protein in color coding are illustrated for each subject. (B) A detailed illustration shows the recognition for each single OLP among the two groups. The red bars indicate the OLPs with a higher frequency of recognition within AIDS patients than elite controllers, while the green bars indicate the OLPs with a higher frequency of recognition within elite controllers. Left $y$ axis indicates the individuals. Right $y$ axis represents the HLA typing results of the individuals. The $x$ axis indicates the nine HIV proteins spanned by the OLPs.

power.

Control of HIV-1 in carriers of the HLA-A*30/B*13/ $C w^{*} 06$ haplotype

We compared the viral loads of individuals with different expressions of HLA class I alleles. Carriers of the
HLA-A $* 30, \mathrm{~B} * 13$ or $\mathrm{Cw} * 06$ alleles were associated with lower viral loads $(P=0.0030,0.0123$ and 0.0039$)$ (Figure $5 \mathrm{~A})$. To determine whether these associations between viral loads and the three HLA-I alleles were independent, we did a linkage disequilibrium analysis using the HLA Analysis Tools supported by Los Alamos HIV 
Table 2 Nine OLPs were found with significantly different frequencies of recognition between elite controllers and AIDS patients

\begin{tabular}{|c|c|c|c|c|c|c|c|}
\hline \multirow[t]{2}{*}{ Protein } & \multirow[t]{2}{*}{ HXB2 location } & \multirow[t]{2}{*}{ OLP sequence } & \multicolumn{2}{|c|}{ Elite controllers } & \multicolumn{2}{|c|}{ AIDS patients } & \multirow[t]{2}{*}{$P$-value } \\
\hline & & & + & - & + & - & \\
\hline \multirow[t]{3}{*}{ Gag } & $141 \rightarrow 158$ & QMVHQPISPRTLNAWVKV & 7 & 7 & 1 & 16 & 0.0109 \\
\hline & $257 \rightarrow 274$ & PVGEIYKRWIILGLNKIV & 5 & 9 & 0 & 17 & 0.0118 \\
\hline & $435 \rightarrow 452$ & GKIWPSHKGRPGNFLQSR & 4 & 10 & 0 & 17 & 0.0318 \\
\hline \multirow[t]{2}{*}{ Vpr } & $9 \rightarrow 26$ & GPQREPYNEWTLELLEEL & 4 & 10 & 0 & 17 & 0.0318 \\
\hline & $17 \rightarrow 34$ & EWTLELLEELKREAVRHF & 5 & 9 & 0 & 17 & 0.0118 \\
\hline Env $^{\#}$ & $579 \rightarrow 596$ & RVLAVERYLKDQQLLGIW & 0 & 14 & 5 & 12 & 0.0482 \\
\hline
\end{tabular}

\#OLPs with a higher frequency of recognition within AIDS patients than elite controllers. The $P$-values are shown before correction for multiple testing.

database (http://www.hiv.lanl.gov), and a HLA-A*30/ $\mathrm{B}^{*} 13 / \mathrm{CW}^{*} 06$ haplotype with a population frequency of 0.2661 was found in this cohort. The infected people expressing HLA-A*30/B*13/Cw*06 presented with a lower median viral load (8 $390 \mathrm{RNA}$ copies $/ \mathrm{ml}$ ) than that of HLA-A*30/B*13/Cw*06-negative subjects (56 200 RNA copies $/ \mathrm{ml})(P=0.0006)$ (Figure $5 \mathrm{~A})$. To determine the contribution of HIV-1-specific CTL response to the association of HLA-A*30/B*13/Cw*06 haplotype with the control of viral replication, we compared the recognition of OLPs within HIV-1 proteins of HLA-A*30/B*13/ $\mathrm{CW}^{*} 06$-positive subjects to that of HLA-A*30/B*13/ $\mathrm{CW} * 06$-negative subjects. In contrast to HLA-A*30/B*13/ $\mathrm{CW}^{*} 06$-positive subjects, significantly more Nef-, Poland Env-OLPs were targeted in the HLA-A*30/B*13/ $\mathrm{CW}^{*} 06$-negative subjects $(P=0.0070,<0.0001$ and $=$ 0.0043 ) (Figure 5B). We also evaluated the associations between viral loads and the number of responsive OLPs in HIV-1 proteins (breadth) in both HLA-A*30/B*13/ $\mathrm{CW}^{*} 06$-positive and -negative subjects. In HLA-A*30/ $\mathrm{B}^{*} 13 / \mathrm{CW}^{*} 06-$ positive individuals, an inverse correlation between viral loads and the breadth of Gag-, Vprand total HIV-1-specific CTL responses was found $\left(r_{\mathrm{s}}=\right.$ $-0.3538,-0.4411$ and $-0.3804 ; P=0.0434,0.0102$ and 0.0290 ), while only a positive correlation between viral loads and the breadth of Env-specific CTL response was found in the individuals without the expression of HLAA*30/B*13/Cw*06 haplotype $\left(r_{\mathrm{s}}=0.4007, P<0.0001\right)$ (Figure 5C and 5D).

\section{Discussion}

The main objective of this study was to characterize virus-specific CTL responses at single peptide level and to investigate their correlations with virus control in a FPD cohort with chronic, untreated HIV-1 clade B' infection. Based on epidemiological investigation, most of these FPDs were infected between 1992 and 1995 through unregulated commercial plasma collection by using contaminated blood collection equipment or by re-infusing pooled erythrocyte concentrations to donors; this practice was discontinued at the end of 1995 [18, 19]. These unusual HIV-infected FPDs represent a unique population to study HIV/AIDS disease progression because the outbreak of HIV-1 infection occurred within a narrowed period of time and the majority of FPDs were infected with very similar virus strains [20,21].

In line with previous studies $[8,22]$, we observed that Gag was the most frequently recognized protein in the HIV-1 B' infection, followed by Pol, Env and Nef. The auxiliary HIV-1 proteins Vif, Vpr, Vpu, Tat and Rev were less frequently targeted by CTL responses, which may be due to their high sequence variability or small size [23]. Seven OLPs from p24 protein of Gag and six OLPs within the central part of Nef were recognized by $>20 \%$ of subjects, indicating these conserved regions are highly immunodominant. To some extent, the discordance of the test peptides and the individual's autologous virus sequence may underestimate the true responses particularly for more variable parts of the HIV products. On the other hand, the persistent stimulation of conservative epitopes would be beneficial for the establishment and maintenance of a pool of memory $\mathrm{T}$ cells, which could be more easily detected by the assay used here. However, HIV-1 Pol, a highly conserved gene product with functional constraints, was targeted by up to $86.71 \%$ subjects at protein level but with a much lower recognition frequency at single peptide level when compared with Gag or Nef. Moreover, Vpr, which is also conserved, was recognized much less frequently than Gag in the present 

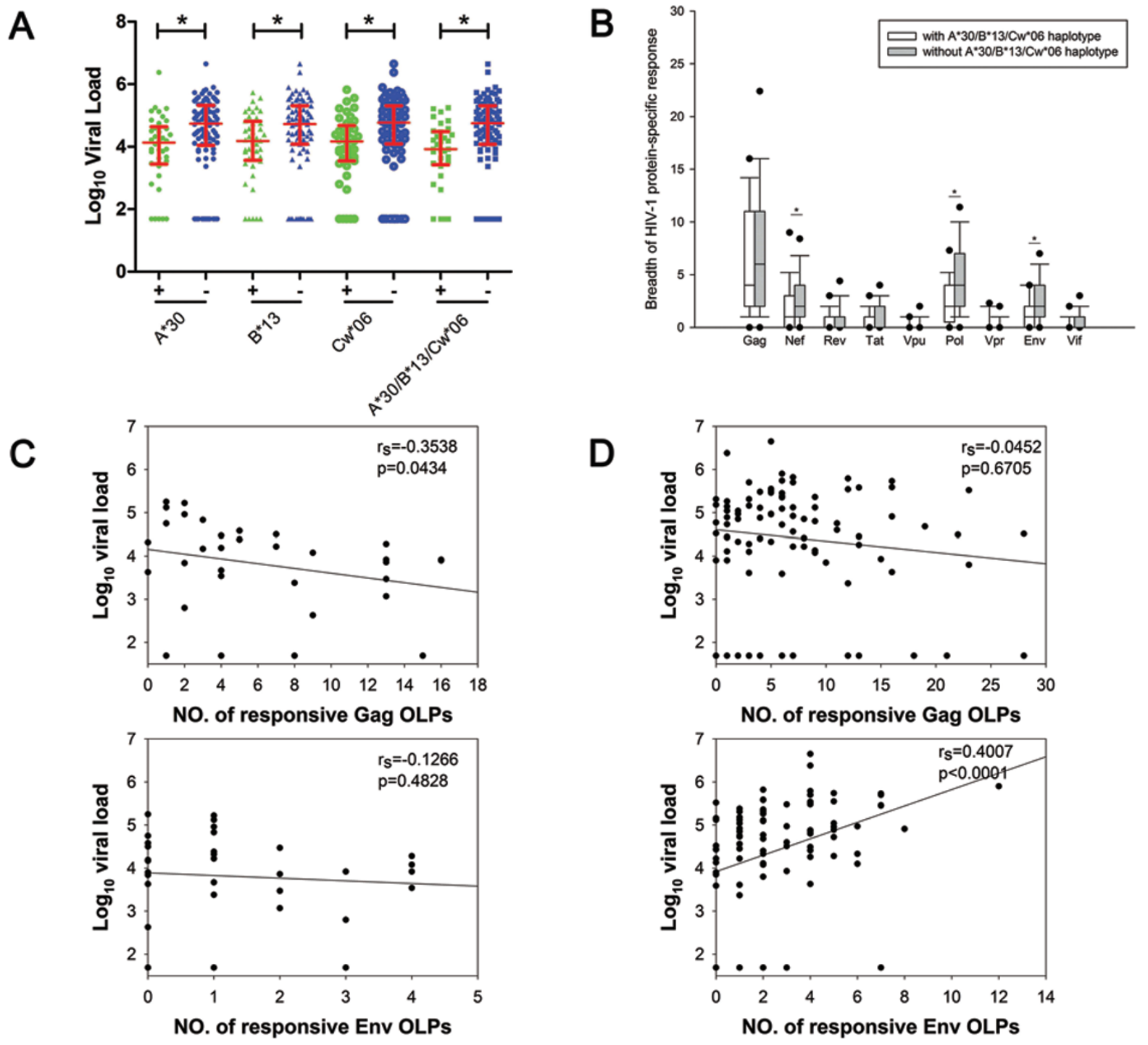

Figure 5 The association of HLA-I molecules with the control of viral replication. (A) The difference of viral loads between the individuals with and without HLA-A*30, $B^{*} 13, C w^{*} 06$ and the HLA-A*30/B*13/Cw*06 haplotype. The red bars show the median with interquartile range. *Represents a $P$-value $<0.05$. (B) Comparison of the HIV-1 protein-specific CTL responses between the individuals with and without the HLA-A*30/B*13/Cw*06 haplotype. The box plots show the median (horizontal line), interquartile range (box), the 10th and 90th percentiles (whiskers), and the 5th and 95th percentiles (points). ${ }^{*}$ Represents a $P$-value $<0.05$. (C) The associations between viral loads and Gag- and Env-specific CTL responses in the individuals with the HLA-A*30/B*13/Cw*06 haplotype. (D) The associations between viral loads and Gag- and Env-specific CTL responses in the individuals without the $\mathrm{HLA}-\mathrm{A}^{*} 30 / \mathrm{B}^{*} 13 / \mathrm{CW}^{*} 06$ haplotype.

study, even when compared with the average recognition frequency of OLPs. Therefore, the conservation of certain proteins perhaps is not sufficient to account for the differential recognition observed. The expression level of HIV proteins may be another factor impacting the recognition of viral proteins by CTL. The conserved Gag/GagPol ratio of 20:1 in infected cells may be favorable to the maintenance of virion infectivity [24, 25]. The higher level of Gag gene expression can trigger a stronger CTL response. Furthermore, the Gag epitopes were derived from incoming virions and presented merely $2 \mathrm{~h}$ post infection, before integration and viral protein synthesis [26]. As a consequence, Gag-specific CTL responses can be easily induced before Nef-mediated MHC class I downregulation. The above reasons may partly explain why Gag was almost always found to be the most immuodominant region in the relevant studies.

The superior role of Gag-specific CTL responses in 
the control of HIV-1 infection has been suggested repeatedly $[5,7,8,16,27]$. In recent investigations, certain epitopes within Gag protein are consistently identified to have a fitness cost when mutation occurs at particular amino acid sites, such as KK10 (KRWIILGLNK) restricted by HLA-B*27 [28], TW10 (TSTLQEQIAW) and IW9 (ISPRTLNAW) restricted by HLA-B*57/5801 [29] and KP9 (KKFGAEVVP) restricted by Mamu$A^{*} 10[30,31]$. The loss of viral replicative capacity of escape variants may contribute to the conservation of HIV-1 proteins with structural and functional constraints like Gag-p24 and Pol-RT [32]. It can be speculated that the CTL responses against these critical epitopes can strongly control HIV replication by applying a strong selection pressure on HIV, which would result in fitness cost. However, in this study, when we compared viral loads to the cumulative breadth and magnitude of IFN- $\gamma$ responses against Gag, no statistically significant correlation was found. Moreover, a statistically significant positive correlation between viral loads and Pol, Env, Nef and total responses was observed. By using the analytical method introduced by Rosario Zuñiga et al. [6], we correlated lower viral loads and higher CD4 counts (slow disease progression) with the relative dominance of Gagspecific CTL responses, while the relative dominance of Pol- and Env-specific CTL responses was correlated with increased viral loads and decreased CD4 counts (fast disease progression). These results confirm the notion that CTL responses to different HIV proteins may have discordant associations with viral loads, and that Gagspecific responses have a superior role in the immune control of HIV infection $[6,8]$. It seems that the CTL response against Pol and Env might not only be ineffective but also harmful for the HIV/AIDS control, as the broad activation of the immune system may awaken the HIV-1 latent reservoir in resting $\mathrm{CD} 4{ }^{+} \mathrm{T}$ lymphocytes and accelerate the spread of HIV in vivo. The upregulation of negative immunoregulatory elements, such as PD-1, CTLA4 and Treg, following systemic activation caused by vast but ineffective CTL responses targeting Pol and Env may also play an important role in the failure of immune control $[33,34]$. In contrast, because of the strong selection pressure that Gag-specific CTL responses apply to the virus as noted above, the replication of virus would be better controlled in patients with a relative dominance of Gag-specific CTL responses. Thus, the balance between "good" responses (e.g., against Gag) and "bad" responses (e.g., against Env or Pol) might be a key mechanism of immune control in chronic HIV-1 infection, which was largely restricted by HLA alleles. On this basis, the discrimination of "good" epitopes and "bad" epitopes and their restriction of HLA alleles will provide more clues to the correlates of immune control. It is foreseeable that the so-called "good" epitopes may tend to cluster in Gag compared to Env or Pol. These findings about the immunodominance of Gag-specific CTL response and its definite association with the relative viral control should be given critical consideration in vaccine design.

Despite its small size, Vpr elicits many crucial effects including inhibition of cell proliferation, induction of apoptosis and modulation of a number of immune molecules $[35,36]$. Like Gag, the structural and functional constraints of Vpr could be in part responsible for determining the induced immune evasion and the replication fitness of HIV-1 [37]. In our study, an inverse correlation between Vpr-specific responses as well as the contribution of Vpr-specific response to total response and viral loads was observed. These data enunciate the comparable effect of Vpr-specific response with Gag-specific response. However, Vpr-specific CTLs were only detected in $16.78 \%$ of the population, which may limit its utility for the design of CTL-based HIV vaccine.

The mechanisms responsible for controlling viral replication in elite controllers remain largely unknown. Several recent studies have associated this control of HIV-1 replication with CTL response, suggesting that a strong $\mathrm{CD}^{+} \mathrm{T}$ cell response with multifunctional activity including the production of multiple cytokines, the proliferative potential and the expression of some unique immune phenotypes is a major mechanism of the control of viral replication [34, 38-40]. Since our study was cross-sectional in design and only a single cytokine was examined, it is difficult for us to precisely elucidate the contribution of CTL response to the full control of HIV replication in these rare elite controllers. However, the potential importance of Gag and Vpr as CTL targets was suggested again in our study with significantly higher relative dominance of Gag- and Vpr-CTL responses observed in elite controllers compared to that of AIDS patients. We also found some OLPs with significantly different frequency of recognition in elite controllers and AIDS patients in the present study. Further studies including HIV sequencing and definition of HLA-restricted optimal epitopes would help to determine whether any escape mutations within the OLPs mainly recognized by elite controllers would result in a fitness cost to HIV.

Some HLA alleles including $\mathrm{B} * 27$ and $\mathrm{B} * 57$ have been demonstrated to have consistent associations with control of HIV/AIDS [3]. However, no such HLA associations with HIV/AIDS control were found in this study. Nevertheless, HLA-A*30/B*13/Cw*06, which is one of the main three-loci haplotypes in the Chinese Han population [41], was shown to be associated with low viral loads in this study. While HLA-B*13 has been pre- 
viously linked with successful HIV/AIDS disease control [42-44], the association of $\mathrm{A}^{*} 30$ and $\mathrm{CW}^{*} 06$ alleles with HIV control has not been reported. Honeyborne et al. [44] suggested that the advantage of HLA-B*13 for HIV-infected individuals is contributed by the targeting of Gag epitopes. This opinion was partly confirmed by our study since an inverse correlation between Gag-specific CTL response and viral loads was observed in the individuals expressing the HLA-A*30/B*13/Cw*06 haplotype. However, we cannot be sure whether HLA-B*13 plays the effective role in the immune control together with the other two alleles, which have a linkage disequilibrium with $\mathrm{B}^{*} 13$.

In summary, this study extends prior efforts and demonstrates that the relative dominance of Gag-specific CTL responses is associated with virus control by analyzing association between HIV-1 protein-specific CTL responses and plasma viral loads or CD4 counts in HIV1-infected FPDs. The contribution of Gag-specific CTL responses to the advantage of HLA-A*30/B*13/Cw*06 haplotype with the control of viral replication further suggests the effectiveness of Gag-specific CTL responses. Further studies about the role of HLA-related viral escape mutations caused by CTL responses and other mechanisms in the control of viral replication in the longterm surviving HIV-1-infected individuals in this cohort may provide useful information to the development and evaluation of HIV vaccines or immunotherapy.

\section{Materials and Methods}

\section{Study subjects}

In all, 143 FPDs infected with HIV-1 clade B' were recruited from Han Chinese at Anhui and Henan provinces, located in central China. All individuals were antiretroviral therapy naive at the time of study. The study subjects consist of 84 males and 59 females, with an average age of 43 years (30-65 years). The medians for CD4 counts and plasma viral loads were 415 cells $/ \mathrm{mm}^{3}(27-$ 1153 cells $\left./ \mathrm{mm}^{3}\right)$ and 27500 copies $/ \mathrm{ml}(<50-4500000$ copies/ $\mathrm{ml})$, respectively. All subjects were diagnosed with HIV infection by using GENELABS HIV BLOT 2.2 Kit (Genelabs) for at least 5 years. The study was approved by the Institutional Review Boards of National Center for AIDS Control and Prevention, the Chinese Center for Disease Control and Prevention, and all subjects provided written informed consent.

\section{Preparation of peripheral blood mononuclear cell}

Peripheral blood mononuclear cells (PBMCs) were prepared from whole blood by density-gradient centrifugation on FicollHypaque (Amersham Biosciences). After being washed twice with Hank's solution, the pellet was resuspended in R10 medium (RPMI 1640 that contained $10 \%$ fetal bovine serum, $100 \mathrm{U} / \mathrm{ml}$ penicillin, $100 \mu \mathrm{g} / \mathrm{ml}$ streptomycin and $2 \mathrm{mmol}$ L-glutamine/l) and counted by microscope. The final concentration of PBMC was adjusted to $1.0 \times 10^{6}$ cells $/ \mathrm{ml}$.

\section{Synthetic HIV-1 peptides}

In all, 413 synthetic 15-20 amino-acid long peptides, overlapping by 10 amino acids and spanning the entire HIV-1 clade B consensus sequence, were synthesized at the Massachusetts General Hospital (MGH) Peptide Core Facility on an automated peptide synthesizer using Fmoc technology.

\section{Elispot assay}

Elispot assays were carried out as described previously [45]. Briefly, PBMC isolated by Ficoll-paque ${ }^{\mathrm{TM}}$ Plus (Amersham Biosciences) density gradient centrifugation were plated in 96-well Elispot plates with polyvinylidene fluoride (PVDF) membrane that had been precoated with $100 \mu$ l of anti-human IFN- $\gamma$ monoclonal antibody $(0.5 \mu \mathrm{g} / \mathrm{ml}$, Mabtech, Stockholm, Sweden). PBMCs were plated at a concentration of 100000 cells/well in a volume of 100 $\mu 1$ of RPMI 1640 medium supplemented with fetal calf serum (10\%), Hepes buffer $(10 \mathrm{mM})$, L-glutamine $(2 \mathrm{mM})$ and penicillinstreptomycin $(50 \mathrm{U} / \mathrm{ml})$. Corresponding clades B peptides were combined into pools of four to six peptides and tested individually when a peptide pool gave a positive response. The final concentration of the peptides in each well was $10 \mu \mathrm{g} / \mathrm{ml}$. Plates were incubated overnight at $37{ }^{\circ} \mathrm{C}, 5 \% \mathrm{CO}_{2}$ and developed the next day as described previously [45]. Wells containing PBMC and medium with PMA/ionomycin or without any peptide were used as positive or negative controls, respectively, and run in triplicate on each plate. To calculate the number of specific $\mathrm{T}$ cell responses, the number of spots in the negative control wells was subtracted from the counted number of spots in each well. Responses were considered positive if there were $>50$ spot-forming cells (SFC) $/ 10^{6}$ PBMC after subtracting background of the mean number of SFC of the three control wells for at least three times.

\section{HLA class I typing}

HLA typing was performed by polymerase chain reaction sequence-specific primers using a commercially available typing kit (PCR-SSP; HLA-ABC SSP Morgan ${ }^{\mathrm{TM}}$ Kits, Texas Biosystems, Inc.).

\section{Viral load testing}

Plasma viral loads was performed using the Amplicor ultrasensitive assay (Hoffman La Roche, Nutley, NJ, USA) according to the manufacturer's instructions, which has a LDL of 50 copies HIV-1 RNA per ml.

\section{CD4 count testing}

CD4 count from EDTA anticoagulated whole blood was performed by using TruCounts (FITC-CD3Ab, PE-CD4Ab, PerCP$\mathrm{CD} 45 \mathrm{Ab}$ and $\mathrm{APC}-\mathrm{CD} 8 \mathrm{Ab})(\mathrm{BD}, \mathrm{USA})$ and FACS Calibur (BD, USA).

\section{Statistical analysis}

Statistical analysis and basic graphical delineation were performed using SigmaPlot 10.0, SigmaStat 3.5 (SPSS Inc.), GraphPad Prism 5 (GraphPad Software Inc.) and Microsoft Excel 2007 (Microsoft Corp.) based on a Spearman's rank correlation, a Mann-Whitney test or a Fisher's exact test, where appropriate; a $P<0.05$ was considered significant. Benjamini and Hochberg False Discovery Rate procedure was performed when correction of multiple testing was needed. Viral-load values below the limit 
of detection (50 RNA copies $/ \mathrm{ml}$ ) were assigned a value of 49 for statistical analysis.

\section{Acknowledgments}

This project has been funded in part by the National Institute of Allergy and Infectious Disease, National Institutes of Health (N01-AI-30024), the China Mega-Project for Infectious Disease (2008ZX10001-010), International Cooperation in Science and Technology from the Chinese Ministry of Science and Technology (2006DFA31510) and the National Nature Science Foundation of China (81172809, 81020108030). We thank Rebecca Armstrong for critical reading of the manuscript.

\section{References}

1 Carrington M, Bontrop RE. Effects of MHC class I on HIV/ SIV disease in primates. AIDS 2002; 16Suppl4:S105-S114.

2 Kawashima Y, Pfafferott K, Frater J, et al. Adaptation of HIV1 to human leukocyte antigen class I. Nature 2009; 458:641645.

3 Carrington M, O'Brien SJ. The influence of HLA genotype on AIDS. Annu Rev Med 2003; 54:535-551.

4 Brumme ZL, Brumme CJ, Carlson J, et al. Marked epitopeand allele-specific differences in rates of mutation in human immunodeficiency type 1 (HIV-1) Gag, Pol, and Nef cytotoxic T-lymphocyte epitopes in acute/early HIV-1 infection. $J$ Virol 2008; 82:9216-9227.

5 Riviere Y, McChesney MB, Porrot F, et al. Gag-specific cytotoxic responses to HIV type 1 are associated with a decreased risk of progression to AIDS-related complex or AIDS. AIDS Res Hum Retroviruses 1995; 11:903-907.

6 Zuniga R, Lucchetti A, Galvan $\mathrm{P}$, et al. Relative dominance of Gag p24-specific cytotoxic T lymphocytes is associated with human immunodeficiency virus control. J Virol 2006; 80:3122-3125.

7 Borghans JA, Molgaard A, de Boer RJ, Kesmir C. HLA alleles associated with slow progression to AIDS truly prefer to present HIV-1 p24. PLoS One 2007; 2:e920.

8 Kiepiela P, Ngumbela K, Thobakgale C, et al. CD8+ T-cell responses to different HIV proteins have discordant associations with viral load. Nat Med 2007; 13:46-53.

9 van Baalen CA, Guillon C, van Baalen M, et al. Impact of antigen expression kinetics on the effectiveness of HIV-specific cytotoxic T lymphocytes. Eur J Immunol 2002; 32:26442652.

10 Tenzer S, Wee E, Burgevin A, et al. Antigen processing influences HIV-specific cytotoxic T lymphocyte immunodominance. Nat Immunol 2009; 10:636-646.

11 Miura T, Brockman MA, Schneidewind A, et al. HLA-B57/ B*5801 human immunodeficiency virus type 1 elite controllers select for rare gag variants associated with reduced viral replication capacity and strong cytotoxic T-lymphocyte [corrected] recognition. $J$ Virol 2009; 83:2743-2755.

12 Schneidewind A, Brockman MA, Yang R, et al. Escape from the dominant HLA-B27-restricted cytotoxic T-lymphocyte response in Gag is associated with a dramatic reduction in human immunodeficiency virus type 1 replication. J Virol 2007;
81:12382-12393.

13 Addo MM, Yu XG, Rathod A, et al. Comprehensive epitope analysis of human immunodeficiency virus type 1 (HIV-1)specific T-cell responses directed against the entire expressed HIV-1 genome demonstrate broadly directed responses, but no correlation to viral load. $J$ Virol 2003; 77:2081-2092.

14 Cao J, McNevin J, Holte S, Fink L, Corey L, McElrath MJ. Comprehensive analysis of human immunodeficiency virus type 1 (HIV-1)-specific gamma interferon-secreting CD8+ T cells in primary HIV-1 infection. J Virol 2003; 77:6867-6878.

15 Betts MR, Ambrozak DR, Douek DC, et al. Analysis of total human immunodeficiency virus (HIV)-specific CD4(+) and CD8(+) T-cell responses: relationship to viral load in untreated HIV infection. J Virol 2001; 75:11983-11991.

16 Novitsky V, Gilbert P, Peter T, et al. Association between virus-specific T-cell responses and plasma viral load in human immunodeficiency virus type 1 subtype $\mathrm{C}$ infection. $J$ Virol 2003; 77:882-890.

17 Pereyra F, Addo MM, Kaufmann DE, et al. Genetic and immunologic heterogeneity among persons who control HIV infection in the absence of therapy. J Infect Dis 2008; 197:563571.

18 Kaufman J, Jing J. China and AIDS - the time to act is now. Science 2002; 296:2339-2340.

19 Zhang W, Hu D, Xi Y, Zhang M, Duan G. Spread of HIV in one village in central China with a high prevalence rate of blood-borne AIDS. Int J Infect Dis 2006; 10:475-480.

20 Su B, Liu L, Wang F, et al. HIV-1 subtype B' dictates the AIDS epidemic among paid blood donors in the Henan and Hubei provinces of China. AIDS 2003; 17:2515-2520.

21 Zhang L, Chen Z, Cao Y, et al. Molecular characterization of human immunodeficiency virus type 1 and hepatitis $\mathrm{C}$ virus in paid blood donors and injection drug users in China. $J$ Virol 2004; 78:13591-13599.

22 Frahm N, Korber BT, Adams CM, et al. Consistent cytotoxicT-lymphocyte targeting of immunodominant regions in human immunodeficiency virus across multiple ethnicities. $J$ Virol 2004; 78:2187-2200.

23 Novitsky V, Cao H, Rybak N, et al. Magnitude and frequency of cytotoxic T-lymphocyte responses: identification of immunodominant regions of human immunodeficiency virus type 1 subtype C. J Virol 2002; 76:10155-10168.

24 Briggs JA, Simon MN, Gross I, et al. The stoichiometry of Gag protein in HIV-1. Nat Struct Mol Biol 2004; 11:672-675.

25 Shehu-Xhilaga M, Crowe SM, Mak J. Maintenance of the $\mathrm{Gag} / \mathrm{Gag}$-Pol ratio is important for human immunodeficiency virus type 1 RNA dimerization and viral infectivity. $J$ Virol 2001; 75:1834-1841.

26 Sacha JB, Chung C, Rakasz EG, et al. Gag-specific CD8+ $\mathrm{T}$ lymphocytes recognize infected cells before AIDS-virus integration and viral protein expression. J Immunol 2007; 178:2746-2754.

27 Edwards BH, Bansal A, Sabbaj S, Bakari J, Mulligan MJ, Goepfert PA. Magnitude of functional CD8+ T-cell responses to the gag protein of human immunodeficiency virus type 1 correlates inversely with viral load in plasma. J Virol 2002; 76:2298-2305.

28 Ammaranond P, Zaunders J, Satchell C, van Bockel D, Cooper DA, Kelleher AD. A new variant cytotoxic T lymphocyte 
escape mutation in HLA-B27-positive individuals infected with HIV type 1. AIDS Res Hum Retroviruses 2005; 21:395397.

29 Leslie AJ, Pfafferott KJ, Chetty P, et al. HIV evolution: CTL escape mutation and reversion after transmission. Nat Med 2004; 10:282-289.

30 Fernandez CS, Stratov I, De Rose R, et al. Rapid viral escape at an immunodominant simian-human immunodeficiency virus cytotoxic T-lymphocyte epitope exacts a dramatic fitness cost. J Virol 2005; 79:5721-5731.

31 Fernandez CS, Smith MZ, Batten CJ, et al. Vaccine-induced T cells control reversion of AIDS virus immune escape mutants. J Virol 2007; 81:4137-4144.

32 Wright JK, Novitsky V, Brockman MA, et al. Influence of Gag-protease-mediated replication capacity on disease progression in individuals recently infected with HIV-1 subtype C. J Virol 2011; 85:3996-4006.

33 Kaufmann DE, Walker BD. PD-1 and CTLA-4 inhibitory cosignaling pathways in HIV infection and the potential for therapeutic intervention. J Immunol 2009; 182:5891-5897.

34 Owen RE, Heitman JW, Hirschkorn DF, et al. HIV+ elite controllers have low HIV-specific T-cell activation yet maintain strong, polyfunctional T-cell responses. AIDS 2010; 24:10951105.

35 Muthumani K, Hwang DS, Dayes NS, Kim JJ, Weiner DB. The HIV-1 accessory gene vpr can inhibit antigen-specific immune function. DNA Cell Biol 2002; 21:689-695.

36 Majumder B, Venkatachari NJ, Srinivasan A, Ayyavoo V. HIV-1 mediated immune pathogenesis: spotlight on the role of viral protein R (Vpr). Curr HIV Res 2009; 7:169-177.

37 Srinivasan A, Ayyavoo V, Mahalingam S, et al. A comprehensive analysis of the naturally occurring polymorphisms in HIV-1 Vpr: potential impact on CTL epitopes. Virol J 2008;
5:99.

38 Saez-Cirion A, Lacabaratz C, Lambotte O, et al. HIV controllers exhibit potent CD8 T cell capacity to suppress HIV infection ex vivo and peculiar cytotoxic T lymphocyte activation phenotype. Proc Natl Acad Sci USA 2007; 104:6776-6781.

39 Betts MR, Nason MC, West SM, et al. HIV nonprogressors preferentially maintain highly functional HIV-specific CD8+ T cells. Blood 2006; 107:4781-4789.

40 Emu B, Sinclair E, Hatano H, et al. HLA class I-restricted T-cell responses may contribute to the control of human immunodeficiency virus infection, but such responses are not always necessary for long-term virus control. J Virol 2008; 82:5398-5407.

41 Yuan F, Sun YY, Luo Y, et al. Haplotype and linkage analysis of HLA-I classical genes in Chinese Han population. Zhongguo Shi Yan Xue Ye Xue Za Zhi 2007; 15:1084-1089.

42 Harrer EG, Bergmann S, Eismann K, et al. A conserved HLA B13-restricted cytotoxic T lymphocyte epitope in Nef is a dominant epitope in HLA B13-positive HIV-1-infected patients. AIDS 2005; 19:734-735.

43 Tang J, Tang S, Lobashevsky E, et al. Favorable and unfavorable HLA class I alleles and haplotypes in Zambians predominantly infected with clade $\mathrm{C}$ human immunodeficiency virus type 1. J Virol 2002; 76:8276-8284.

44 Honeyborne I, Prendergast A, Pereyra F, et al. Control of human immunodeficiency virus type 1 is associated with HLAB*13 and targeting of multiple gag-specific CD8+ T-cell epitopes. J Virol 2007; 81:3667-3672.

45 Lichterfeld M, Yu XG, Cohen D, et al. HIV-1 Nef is preferentially recognized by CD8 $\mathrm{T}$ cells in primary HIV-1 infection despite a relatively high degree of genetic diversity. AIDS 2004; 18:1383-1392.

(Supplementary information is linked to the online version of the paper on the Cell Research website.) 